Methods This is a retrospective and descriptive study including all full-term neonates deceased before leaving hospital. The Data were collected between January 2016 and December 2018 from the neonatology department of Sfax's University Hospital HédiChaker (Tunisia).

The International Classification of Diseases (ICD) codes' have been followed to classify death's diagnosis.

Results During the study's period 93 term newborns had died in the hospital. $68 \%$ were born by caesarean section and $24 \%$ had a low birth weight. The death occurred during the first week of life in $74 \%$ of cases. The leading causes of death were congenital malformations (56\%) and perinatal asphyxia (22\%). Only 2 cases were deceased by other causes than maternofetal complications.

Conclusion The prenatal diagnosis of fetal anomalies and the quality of the childbirth monitoring are the main factors associated to the hospital term neonatal death. Further research is required to understand causes of full-term's mortality so its incidence and effects can be reduced.

\section{P635 IMPACT OF ANTENATAL CORTICOSTEROID THERAPY ON THE NEONATAL PROGNOSIS OF PRETERM INFANTS BORN BEFORE 33 WEEKS OF AMENORRHEA}

Ilyes Regaieg, Amel Ben hmed, Manel Charfi, Chiraz Regaieg*, Amira Bouraoui, Nedia Hmida, Ridha Regaieg, Afef Ben Thabet, Abdellatif Gargouri. Department of Neonatology, Hedi Chaker Hospital, Sfax, Tunisia

\subsection{6/archdischild-2019-epa.966}

Introduction Antenatal corticosteroid treatment is widely proved to be efficient in reducing hyaline membrane disease and prematurity complications. However, the effects of administering a course of corticosteroids to the mother prior to anticipated preterm birth on fetal and neonatal morbidity and mortality are still unclear. Therefore, the objective of this researchis to assess theses effects on preterm infants born before 33 weeks of amenorrhea.

Patients and methods In this retrospective and descriptive study, we have considered all premature infants who were born before 33 weeks and hospitalized in the neonatology department of Sfax's University Hospital Hedi Chaker (Tunisia) between 1 January 2014 and 31 December 2016.

The short-term prognosis was compared for preterm newborns according to whether antenatal corticotherapy was performed or not.

Results During the period of research, 565 premature infants born before 33 weeks of amenorrhea were hospitalized. Thus the rate of the early prematurity was $1.9 \%$.

Antenatal corticosteroids were performed in $76.1 \%$ of cases.

The frequency of hyaline membrane disease was $22.8 \%$ in the presence of this corticosteroid treatment against $44.4 \%$ when it was not performed.

The incidence of intracranial haemorrhage and ulcerative necrotizing enterocolitis was higher in premature infants who did not receive antenatal corticosteroid therapy.

The overall hospital mortality was $25.1 \%$ in this series. It was significantly higher in the absence of antenatal corticosteroid therapy (39.3\% versus $20.7 \%)$.
The main causes of death were immaturity, hyaline membrane disease, nosocomial infections and intracranial haemorrhage.

Conclusion The antenatal corticosteroid therapy has a great impact on mortality of preterm infants born before 33 weeks. Our findings highlight the value antenatal corticotherapy to reduce morbidity and mortality in these premature infants.

Follow-up studies into childhood and adulthood, particularly in the psychomotor and neuro-development is needed and it would be especially relevant to explore this finding in adequately powered prospective trials.

\section{P636 AN UNUSUAL CAUSE OF GLUTEAL SWELLING.... CONGENITAL RECTAL DUPLICATION CYST}

${ }^{1}$ Qasim Mahmood, 'Uzair Shabbir*, ${ }^{2}$ Brian Sweeney, ${ }^{2}$ Maureen O'Sullivan, ${ }^{1} T a h a$ Yousif. ${ }^{1}$ University Hospital Kerry, Tralee, Ireland; 'Our Lady's Children Hospital, Crumlin, Dublin, Ireland

\subsection{6/archdischild-2019-epa.967}

Rectal Duplication (RD) is a very rare phenomenon with only around 100 cases reported.They make up a merely 5\% of all intestinal duplication's. Most of the rectal duplication's are located in the retro rectal space.

Presenting symptoms include intestinal obstruction,bladder outlet obstruction,dysuria,pelvic pain,mucous or purulent drainage from the rectum or perianal fistula,rectal bleeding from the presence of heterotopic gastric mucosa,constipation and rectal prolapse. RDs are mostly diagnosed with ultrasound and MRI.Treatment of choice in majority of cases is surgical resection.

We report a very unusual case of congenital rectal duplication cyst in a newborn.A male baby was delivered by emergency C-section for maternal pre-eclampsia at 39 weeks gestation with birth weight of $3.9 \mathrm{~kg}$.Initial clinical review revealed a large,shiny,dark bluish cystic type lesion on the right buttock.Ultrasound scan of the right buttock showed a large deep cystic lesion measuring $3 \mathrm{~cm}$ in diameter in the right gluteal region.At 16 hours of age,the lesion burst through one centimeter opening discharging copious amount of liquid meconium,resulting in the collapse of the swelling.As a result,he received fluid resuscitation,feeds were withheld and he was started on antibiotics for suspected sepsis.

MRI pelvis showed a large fluid filled tubular structure in the presacral space displacing both the rectum and bladder. The imaging also showed dilatation of the upper tracts in relation to both kidneys with displaced and distended bladder. Surgery showed rectal duplication cyst sharing a common wall with the rectum,however,there was no direct connection with the rectal lumen and was successfully excised.

The biopsies revealed degenerative and necrotic tissue which was unusual.The presence of meconium could be attributed to the presence of a direct connection with the rectal cavity at some stage of the development.

The baby was discharged home post surgery and made full recovery without any complications.

To our knowledge,this case is the first to present with gluteal swelling and subsequent rupture yielding liquid meconium. 